\title{
Le château de Châteauneuf (Côte-d'Or) : origines, évolution et réutilisation
}

Mémoire de Master 2 sous la direction de Denis Cailleaux, université de Bourgogne. Soutenu le 7 juin 2016

\section{Thomas Vergine}

\section{(2) OpenEdition}

Journals

Édition électronique

URL : https://journals.openedition.org/cem/14680

DOI : $10.4000 /$ cem. 14680

ISSN : 1954-3093

Éditeur

Centre d'études médiévales Saint-Germain d'Auxerre

Référence électronique

Thomas Vergine, « Le château de Châteauneuf (Côte-d'Or) : origines, évolution et réutilisation », Bulletin du centre d'études médiévales d'Auxerre | BUCEMA [En ligne], 21.1 | 2017, mis en ligne le 18 septembre 2017, consulté le 22 septembre 2022. URL : http://journals.openedition.org/cem/14680 ; DOI : https:// doi.org/10.4000/cem. 14680

Ce document a été généré automatiquement le 22 septembre 2022.

\section{c) (i) (8)(2)}

Creative Commons - Attribution - Pas d'Utilisation Commerciale - Partage dans les Mêmes Conditions 4.0 International - CC BY-NC-SA 4.0

https://creativecommons.org/licenses/by-nc-sa/4.0/ 


\section{Le château de Châteauneuf (Côte- d'Or) : origines, évolution et réutilisation}

Mémoire de Master 2 sous la direction de Denis Cailleaux, université de Bourgogne $^{1}$. Soutenu le 7 juin 2016

\section{Thomas Vergine}

"Quels sont les principaux monuments d'architecture civile et militaire de l'époque du moyen-âge (...) que possède encore la Bourgogne (...)?» Quand M. Canat pose cette question au Congrès archéologique de France, qui se déroule à l'été 1852 à Dijon, la première intervention est celle de M. Foisset qui cite d'emblée Châteauneuf comme «la plus belle construction féodale du pays $»^{2}$. Même si cette affirmation reste à nuancer, le château de Châteauneuf n'en demeure pas moins un riche témoin de l'architecture castrale, aujourd'hui

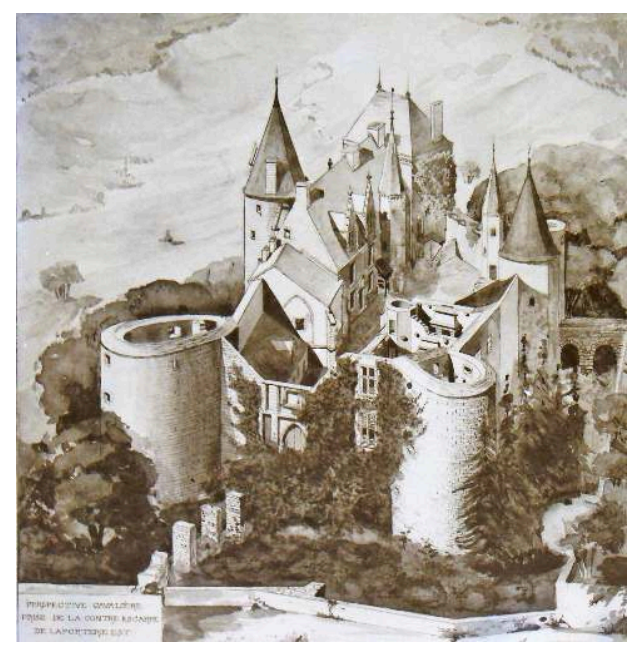
conservé en bonne partie. Il présente le net avantage de n'avoir pas subi de démantèlement au XvII siècle, comme ce fut le cas de la plupart des châteaux bourguignons tenus par des opposants d'Henri IV ${ }^{3}$ (Vitteaux, Brémur, etc.) et n'a pas eu à souffrir de l'expansion urbaine du petit village de Châteauneuf au xix siècle, qui aurait pu compromettre son intégrité. De fait, cet imposant château du sud de l'Auxois a pu attirer la curiosité des érudits locaux et des savants régionaux, depuis Claude Courtépée jusqu'à Anatole de Charmasse ${ }^{4}$ - pour les plus connus - en passant par le curé de la paroisse et le propriétaire du château au début du Xx $x^{e}$ siècle $^{5}$. 
Dans l'ensemble, l'histoire du château de Châteauneuf - et de la seigneurie associée est assez bien connue, complétée par l'apport régulier de nouvelles données et méthodes: approche castellologique ( $\mathrm{CeCaB})$, mémoires universitaires, études dendrochronologique et archéologique ponctuelles ${ }^{6}$. Le château ne cesse pas pour autant d'être l'enjeu de questionnements toujours plus poussés que suscitent des initiatives récentes.

\section{Densification du maillage castral et édification du château neuf}

Le nombre de castra présents sur le territoire de la Côte-d'Or s'avère assez réduit avant l'an Mil, environ une vingtaine de sites, parmi lesquels on citera des enceintes de l'Antiquité tardive (Til-Châtel, Beaune) ou des forteresses du haut Moyen Âge (Grignon, Vergy). Et s'il a un temps paru judicieux de faire appel à l'expression de "premier réseau castral » pour dénommer ces forteresses antérieures aux années 1050, opposées à un " second réseau castral » regroupant la vague de constructions de châteaux entre le milieu du XI ${ }^{\mathrm{e}}$ et le $\mathrm{XIII}^{\mathrm{e}}$ siècle, il semble dorénavant plus pertinent de considérer que la Bourgogne a connu un mouvement lent, mais quasi ininterrompu, de fondations castrales entre le $\mathrm{III}^{\mathrm{e}}$ et le $\mathrm{x}^{\mathrm{e}}$ siècle ${ }^{7}$, puis, à partir des années 1050, une augmentation exponentielle du nombre de châteaux, sans qu'il y ait nécessairement une rupture entre ces deux phases (fig. 1).

Fig. 1 - Carte des castra mentionnés

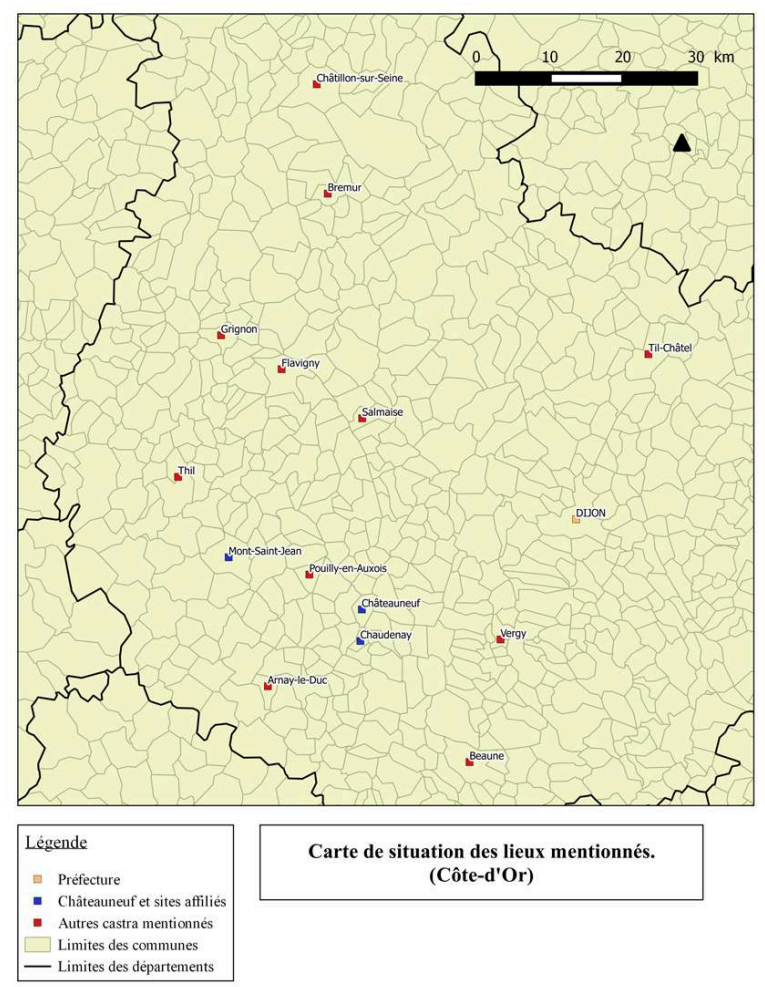

T. Vergine 
marquée. Par ailleurs, on se base trop souvent sur la première mention d'un château, parfois bien éloignée de la date de fondation : à Salmaise, par exemple, les vestiges de la première fortification du château sont datables entre le vil et le $\mathrm{x}^{\mathrm{e}}$ siècle ${ }^{8}$, alors que les premières mentions textuelles du château ne sont que du début du XI ${ }^{\mathrm{e}}$ siècle ${ }^{9}$.

5 Toutefois, il semble certain qu'à partir de l'an Mil intervient une densification du maillage castral dans les interstices du réseau assez lâche des premières forteresses. Plusieurs facteurs méritent attention lorsque l'on tente d'expliquer cette multiplication des châteaux. Un contexte favorable d'expansion démographique et économique aux alentours de l'an Mil est forcément à prendre en compte. Il serait cependant réducteur de limiter à cette seule cause la multiplication des forteresses. À mon avis et pour reprendre Florian Mazel, le premier et le plus important de ces facteurs tient à la segmentation régulière des titres et des honneurs du père à ses fils, en dépit d'une pratique successorale favorisant le fils aîné ${ }^{10}$. Un tel modèle lignager, exclusif aux dynasties princières avant l'an Mil, se vulgarise au $\mathrm{xI}^{\mathrm{e}}$ siècle dans les lignages seigneuriaux ${ }^{11}$. De fait, les familles qui ont connu un accroissement foncier ou de fortune procèdent à un partage ou, en tout cas, à une meilleure dotation des cadets, qui s'établissent alors sur les domaines dont ils ont hérité pour prendre possession ou édifier de nouvelles résidences fortifiées. C'est pourquoi on peut estimer que la plupart de ces châteaux postérieurs à l'an Mil, du moins à l'origine, étaient pour une bonne part des forteresses secondaires ou de périphérie, plus modestes que la forteresse principale. Ces châteaux sont parfois édifiés par les grands seigneurs pour couvrir l'approche de leur propre forteresse, voire pour assurer leurs prétentions sur des terres contestées $^{12}$ ou même pour tirer profit d'une ressource particulière ${ }^{13}$. D'autres facteurs sont évidemment à relever. L'évolution politique conserve d'ailleurs un certain poids dans ce mouvement, en particulier le passage d'un royaume centrifuge à des ensembles politiques centripètes.

$6 \mathrm{Au}$ sein de la vaste série de châteaux émergeant à partir $\mathrm{du} \mathrm{xI}^{\mathrm{e}}$ siècle, celui de Châteauneuf ne prend forme que dans la seconde moitié du XII ${ }^{\mathrm{e}}$ siècle, c'est-à-dire assez tardivement. Ce n'est, à l'origine, qu'une forteresse secondaire ayant pour but de couvrir les abords du château de Chaudenay (à $5 \mathrm{~km}$ au sud), qui semble lui-même lié d'une manière ou d'une autre à la vaste seigneurie de Mont-Saint-Jean ${ }^{14}$. Le site d'implantation du nouveau château est d'ailleurs révélateur de sa fonction : l'éperon forme un rameau qui se sépare du haut plateau pour descendre en pente douce vers la vallée de la Vandenesse, tout en présentant un dénivelé de $100 \mathrm{~m}$ entre le château ( $465 \mathrm{~m}$ ) et la plaine $(365 \mathrm{~m})$. L'intérêt majeur du site est qu'il surveille un nœud routier important situé à Pouilly-en-Auxois - où passe d'ailleurs l'A6 -, qui ouvre la voie en direction du nord-ouest - en remontant l'Armançon ou le Serein -, vers Dijon à l'est, en rejoignant le col de Sombernon, ou encore vers le sud-ouest, en direction d'Arnay-leDuc, Autun ou même Beaune.

7 Dans un premier temps, le château est administré par un prévôt du sire de Chaudenay ${ }^{15}$, jusqu'au décès de ce dernier en 1179-1181. Tandis que l'aîné, Guy de Chaudenay, hérite du château familial éponyme, le cadet, Jean, se voit accorder Châteauneuf. Il adopte désormais le toponyme du domaine hérité comme nom patronymique, de manière à distinguer la branche cadette de celle de son frère aîné, et il fait de cette forteresse secondaire sa résidence et le chef-lieu d'une nouvelle seigneurie disposant d'un détroit moins étendu que l'ancien pôle familial, mais avec les prérogatives seigneuriales du 
même ordre. En outre, dès $1181^{16}$, Jean $\mathrm{I}^{\text {er }}$ de Châteauneuf utilise systématiquement ce topo-lignage, accompagné du qualificatif dominus, pour indiquer de fait son statut de seigneur sur le domaine castelnovien, qui lui appartient désormais. Au moment de la succession, le château n'a pas l'apparence de l'édifice actuel, qui ne conserve aucun élément visible remontant à cette période.

\section{L'évolution du château (XIII $-\mathrm{XV}^{\mathrm{e}}$ siècle)}

La construction du château s'achève très vraisemblablement au début du XIII ${ }^{\mathrm{e}}$ siècle, sous l'égide de son premier seigneur, Jean de Châteauneuf. Ce second état du château semble adopter le plan classique d'une large enceinte oblongue, qui se développe sur la pointe du plateau calcaire, se divisant en deux cours distinctes, une cour basse et une cour haute, plus restreinte, dans la partie la plus élevée de l'enceinte ${ }^{17}$. Ce type de plan est assez récurrent pour les enceintes fortifiées régionales, par exemple à Mont-SaintJean et Grancey. La portion nord-ouest de l'actuelle courtine préserve certains éléments de la première enceinte, dont les fondations en grand appareil et la porte à pont-levis à bascule inscrite dans une tour carrée. Cependant, l'élément le plus important et le mieux conservé demeure l'imposant donjon quadrangulaire - $10 \times 12 \mathrm{~m}$, pour une hauteur de $25 \mathrm{~m}$. Au moment où le modèle philippien se développe pour l'architecture castrale, on prend à Châteauneuf le parti d'édifier un donjon sensiblement similaire à ceux voisins de Soussey-sur-Brionne et Gevrey-Chambertin, tous du XIII ${ }^{\mathrm{e}}$ siècle $^{18}$, ce que corroborent d'ailleurs divers éléments typo-chronologiques, stylistiques et architecturaux ${ }^{19}$ (fig. 2).

Fig. 2 - Pont-levis à bascule et donjon en arrière-plan

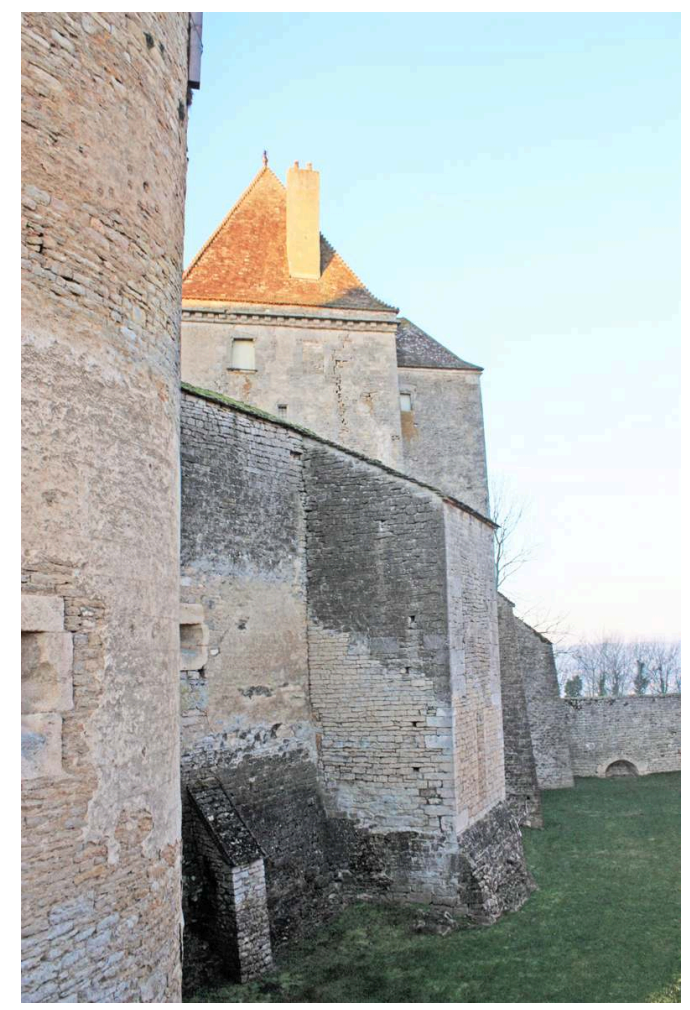

Première moitié du xIII' siècle

Cl. T. Vergine 
9 Mais si le château n'adopte pas le principe d'architecture philippien dès son édification, les sires de Châteauneuf introduisent finalement ce modèle à la fin du XIII siècle par l'adjonction de deux tours circulaires sur la courtine de la cour haute : l'une à l'angle sud, l'autre à l'angle est (fig. 3).

\section{Fig. 3 - Porterie nord}

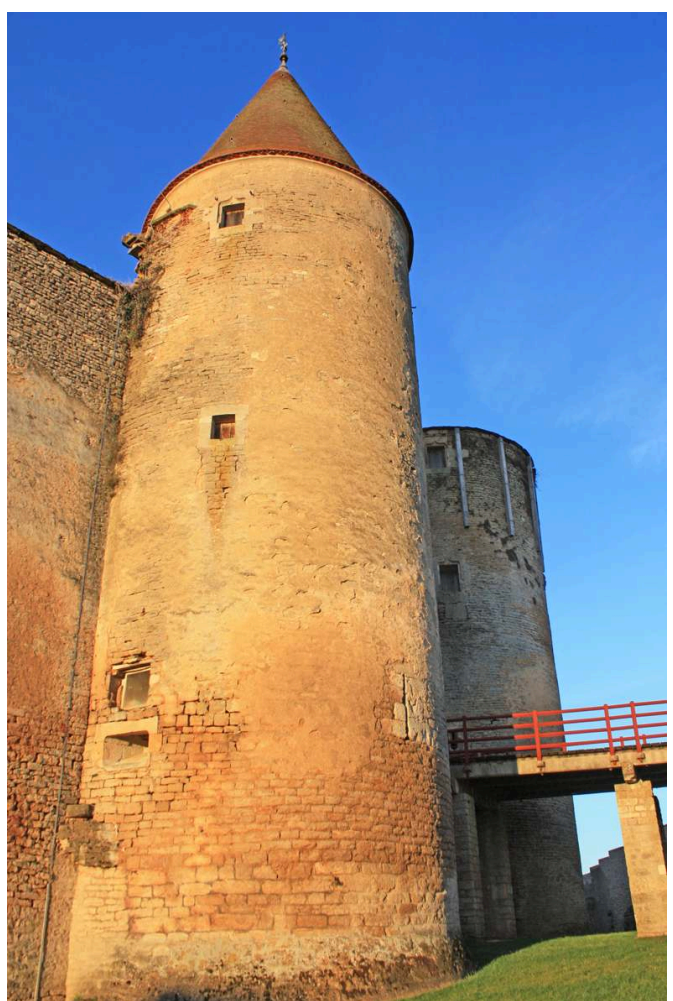

La tour au premier plan est de la seconde moitié du xIII ${ }^{e}$ siècle, celle en arrière-plan est de la fin du $x v^{e}$ siècle.

Cl. T. Vergine

10 Le but est de flanquer l'enceinte pour doter le château d'un système de défense actif. Parallèlement, c'est aussi à la fin du XIII ${ }^{e}$ siècle que la chapelle Saint-Jean est édifiée dans la cour basse du château ${ }^{20}$ (fig. 4). 
Fig. 4 - Plan schématique du château et évolution

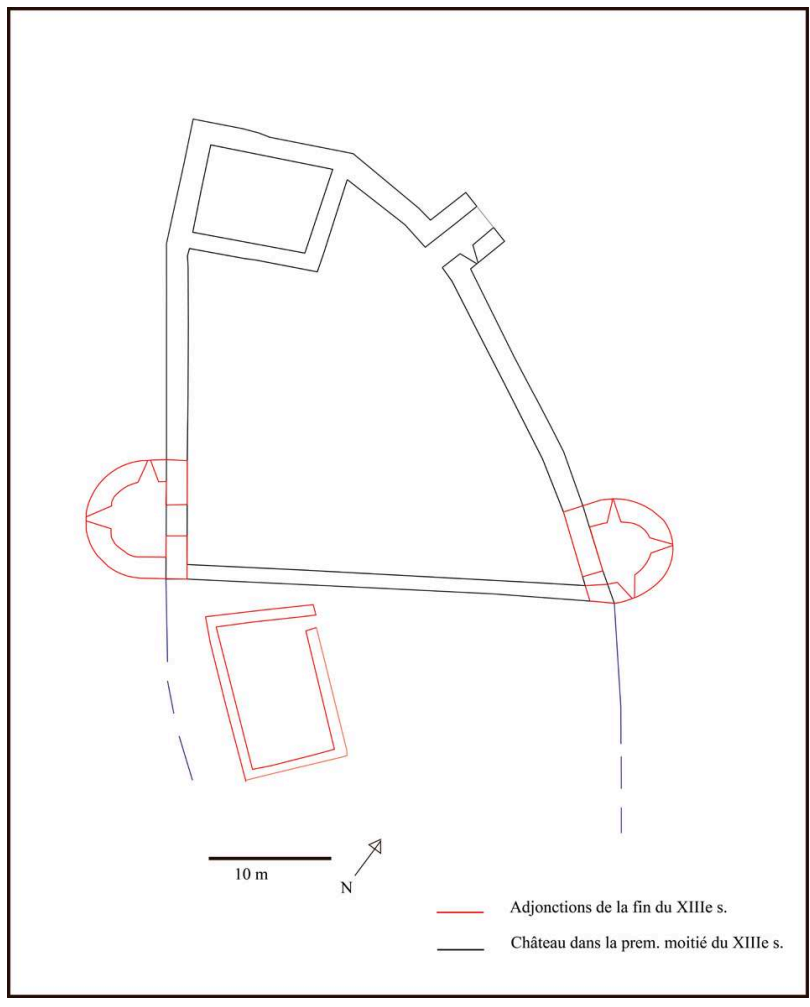

XIII ${ }^{\mathrm{e}}$ siècle

T. Vergine

11 Peu de modifications semblent avoir été apportées au château au cours du XIV siècle. C'est réellement dans la première moitié du siècle suivant que des transformations modifient profondément la structure initiale de la vaste enceinte castrale divisée en deux cours, l'une pour les communs et la chapelle, l'autre, plus fortement défendue, pour le donjon et le logis seigneurial ${ }^{21}$. Cette phase voit l'agrandissement de la cour haute sur une longueur d'une trentaine de mètres vers l'est, au détriment de la cour basse, désormais séparée de sa voisine par un fossé creusé dans le substrat calcaire. De fait, la cour basse, en partie amputée, n'est presque plus défendue et ne paraît plus vraiment intégrée à l'emprise de la forteresse. En revanche, la portion nouvellement intégrée à la cour haute est ceinturée d'une haute courtine flanquée de deux nouvelles tours massives en fer à cheval, elles-mêmes reliées par un nouveau mur, percé à la fin du même siècle pour aménager une porte à pont-levis (fig. 5). 
Fig. 5 - Schéma axonométrique illustrant les modifications de la première moitié du xve siècle
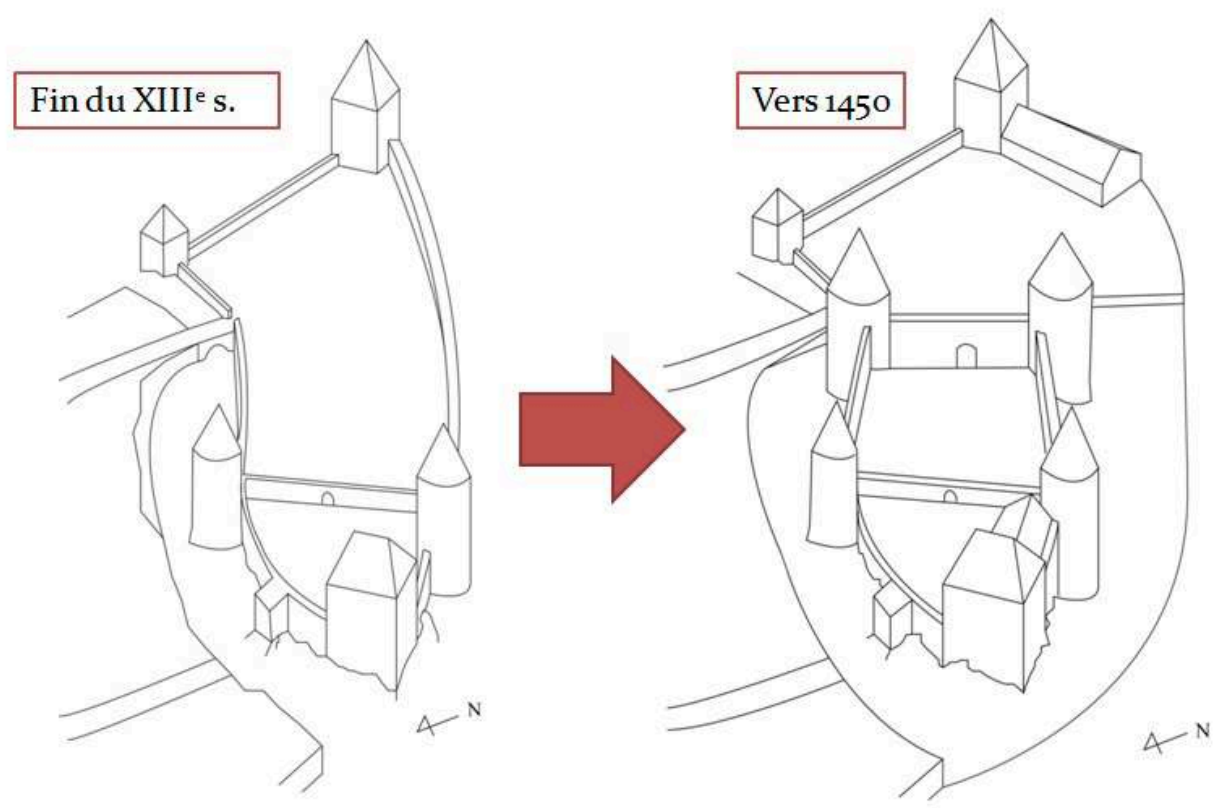

T. Vergine

C'est également à cette phase qu'il semble falloir attribuer l'aménagement des canonnières.

La dernière campagne importante de modifications, voire de reconstruction du château médiéval, débute en $1457^{22}$. Le premier lignage seigneurial à peine éteint - après la fin rocambolesque de la dame de Châteauneuf ${ }^{23}$-, le château revient à Philippe Pot, par l'entremise du duc de Bourgogne. Tout juste après en être devenu propriétaire, le chancelier ducal se lance dans d'importants travaux, sous l'influence architecturale des hôtels particuliers du $\mathrm{xv}^{\mathrm{e}}$ siècle. Cette campagne est marquée par la reconstruction et par l'embellissement du Grand Logis, conjointement au remaniement de la courtine sud. Elle voit également la (re-)construction d'un second logis dans l'angle opposé de la cour - le logis Philippe Pot -, la réalisation d'un nouveau décor pour la chapelle castrale et le remaniement de la courtine orientale pour l'aménagement d'une nouvelle porterie à pont-levis, partiellement inachevée et qui mène à l'église de l'ancienne cour basse. Outre ces modifications, qui sont principalement d'ordre résidentiel, le château renforce ses défenses : la construction d'une seconde porterie au nord, accostée d'une tour de flanquement supplémentaire, le rehaussement des courtines et une meilleure desserte de ces dernières par l'adjonction de vis d'escalier hors œuvre aux principales tours en témoignent (fig. 6). 
Fig. 6 - Plan schématique du château et évolution

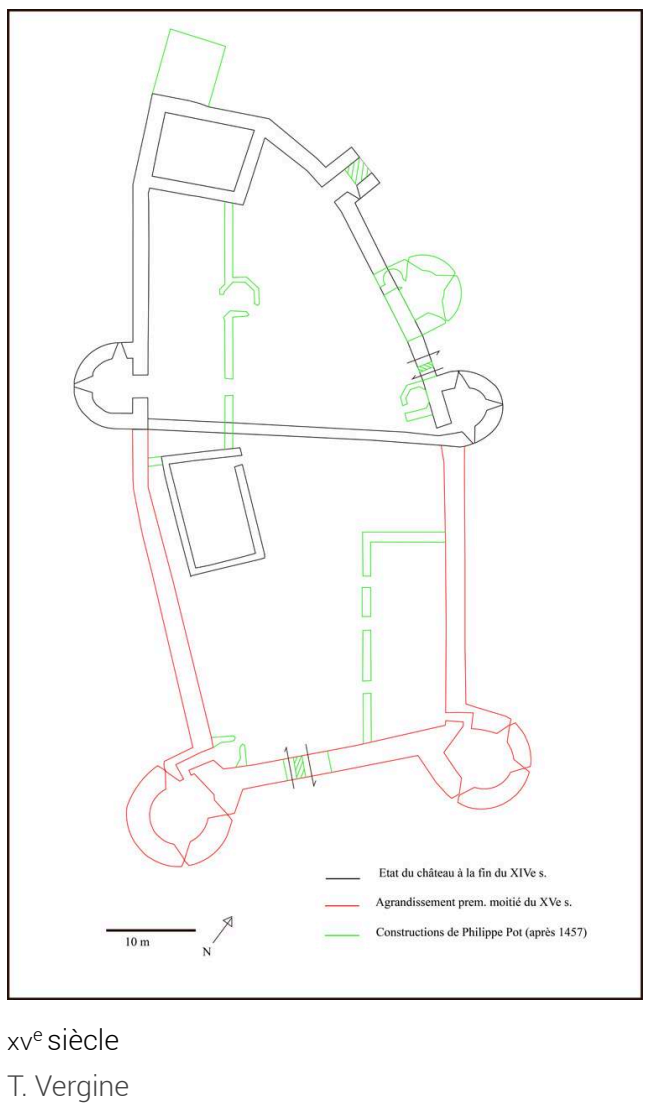

En somme, sans que son caractère défensif soit délaissé, le château est doté d'un complexe résidentiel plus conséquent, richement décoré et orné à l'image d'autres forteresses similaires, selon une dynamique de l'époque qui tend à transformer les forteresses en résidences particulières.

\section{Après le Moyen Âge, quelle utilité ?}

Si l'action de Philippe Pot à Châteauneuf a permis de fortifier et d'embellir le château d'un riche complexe résidentiel, après le décès de ce grand personnage (1493), le site devient définitivement une résidence secondaire pour ses propriétaires successifs, dont les plus connus sont les Montmorency et les Vienne. Cependant, le château ne perd pas pour autant tout intérêt stratégique, car, à compter de 1566, le Calvinisme s'implante et fait de rapides progrès malgré une forte résistance catholique. Ceci entraîne le saccage de villes (Mâcon, Chalon-sur-Saône) et d'abbayes - Moutiers-Saint-Jean, Saint-Martin d'Autun -, ainsi qu'une bataille près d'Arnay-le-Duc à la fin de juin 1570. Après un calme très relatif, l'accession d'Henri de Navarre au trône de France (1589) provoque de nouveaux troubles en Bourgogne, notamment parce que le chef de la Ligue, Charles de Lorraine, gouverneur de Bourgogne, a fait de cette province un bastion de son parti, ralliant de nombreux seigneurs bourguignons, dont Charles de Montmorency, entre autre baron de Châteauneuf, qui commande à son châtelain de mettre le site en état de défense. 
16 Le château de Châteauneuf, place forte des Ligueurs, est assiégé une première fois en 1592 par le marquis de Mirebeau - de la faction royaliste de Bourgogne -, sans succès. Quand la Ligue se rallie à la légitimité d'Henri IV, après son abjuration et son sacre à Chartres en février 1594, seul Charles de Lorraine reste révolté, soutenu par les troupes de Philippe II d'Espagne. Le 3 juin 1595, la victoire de Fontaine-Française anéantit pourtant les derniers espoirs des Ligueurs et le sire de Biron, maréchal du roi, soumet alors les dernières villes et châteaux bourguignons qui résistaient encore, notamment Beaune puis Châteauneuf, devant lequel il met le siège en septembre 1595. Le sire de Villeneuve capitule alors, en novembre, sous la pression des habitants et en échange d'une forte rançon ${ }^{24}$. Le château reste, malgré cela, inexpugnable.

À partir de ce moment, le château devient à proprement parler « inutile ». Il abandonne sa fonction principale en perdant son attrait stratégique et on pourrait presque affirmer qu'il perd sa fonction résidentielle, car ses propriétaires successifs n'en font usage que ponctuellement. Force est de constater que l'on parvient au même bilan pour la majeure partie des forteresses médiévales de Bourgogne ${ }^{25}$.

Échappant au démantèlement et à l'abandon total, le château occupe désormais une nouvelle fonction: il devient au XIX ${ }^{\mathrm{e}}$ siècle l'archétype du "château féodal »; des travaux sont menés depuis son classement au titre des Monuments historiques en 1894, sous les soins attentifs de l'architecte Charles Suisse (1846-1906) ; il sert également de modèle pour la restauration d'autres édifices médiévaux - Rochepot, le Palais des ducs à Dijon, etc. Il est même utilisé comme entrepôt pour abriter les pièces les plus précieuses et les plus fragiles des musées de Dijon au cours de la seconde guerre mondiale. Enfin, avec les récentes restaurations, son ouverture au tourisme et sa mise en valeur, le château ainsi que son village connaissent un attrait, non plus défensif mais culturel (fig. 7). 
Fig. 7 - Perspective cavalière du château de Châteauneuf

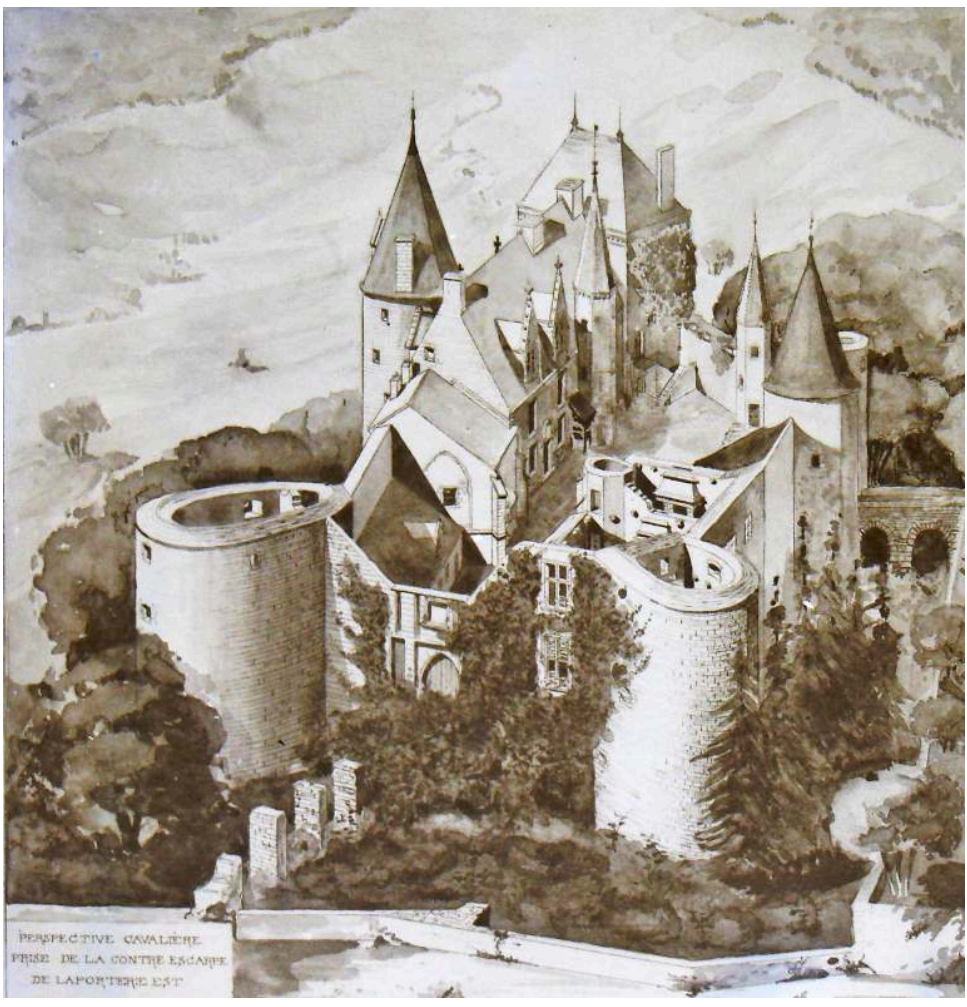

Dessin et aquarelle de Charles Suisse, début du $x x^{e}$ siècle Archives départementales de la Côte-d'Or, 11 Fi 46

Reçu : 2 février 2017 - Accepté : 29 juin 2017

\section{NOTES}

1. Le titre complet du mémoire est Châteauneuf (21) : morphogenèse et évolution d'une agglomération d'origine castrale (XII ${ }^{e}-\mathrm{XVI}^{e}$ siècle), ce texte en est une présentation partielle, centrée sur le château.

2. Congrès archéologique de France, $X I X^{e}$ session, Dijon, 1852, Paris, 1853, p. 75-76.

3. Archives départementales de la Côte-d'Or [ADCO] : C 3075.

4. C. CourTÉPÉE, Description générale et particulière du duché de Bourgogne, t. 6, Dijon, 1780, p. 84-90 ; A. DE CHARMASSE, «Rapport à la Société Éduenne sur l'excursion faite à Châteauneuf et à Commarin le 7 août 1913 ", Mémoires de la Société Éduenne, 41 (1913), p. 243-251.

5. M. LANDROT, Châteauneuf et Vandenesse, Dijon, 1927 ; A. DE VOGÜÉ, « Châteauneuf, son château, ses seigneurs. 1175-1802 », Mémoires de la Société Éduenne, 41 (1913), p. 253-274.

6. C. Perrault, Châteauneuf-en-Auxois (Côte-d'Or). Datation par dendrochronologie des plafonds et des charpentes du château, C.E.D.R.E., Besançon, 2016 ; E. LABORIER, Châteauneuf (21), le château. Rapport de diagnostic archéologique, DRAC/SRA Bourgogne, Dijon, 2002 ; S. AUMARD, Châteauneuf-en-Auxois (21), église Saint-Philippe-et-Saint-Jacques. Études des combles, DRAC/SRA Bourgogne, Dijon, 2011. 
7. Du moins, c'est ce que semblent mettre en avant les données connues sur les castra tardoantiques (Arnay, Avallon, Brémur) et du haut Moyen Âge (Flavigny, Châtillon), suivis par l'apparition de nouveaux châteaux dès la fin du xe siècle (Thil, Pouilly-en-Auxois, etc.).

8. M. Provost, Carte archéologique de la Gaule, t. 21/3 (La Côte-d'Or), Paris, 2009, p. 163-167.

9. ADCO : $01 \mathrm{H} 1767$.

10. F. MAZEL, Féodalités (888-1180), Paris, 2014, p. 448.

11. D. B ARTHÉLEMY, Nouvelle histoire de la France médiévale, t. 3 (L'ordre seigneurial, $\mathrm{XI}^{e}$-XII ${ }^{e}$ siècle), Paris, 1990, p. 31.

12. J. RICHARD, Histoire de la Bourgogne, Toulouse, 1988, p. 138-139.

13. M. MANGin et al., Mines et métallurgie chez les Éduens, Besançon, 1992 : exemple de la motte de Crépy, qui est entourée d'un important réseau de fossés d'extraction et de site de réduction du fer.

14. T. VERGINE, Châteauneuf(21)..., op. cit., t. 1, p. 87-88.

15. ADCO : 11 H 251, 12 H 106.

16. ADCO : $11 \mathrm{H} 251$.

17. L'ensemble mesure environ $130 \times 30 \mathrm{~m}$, avec une cour haute s'apparentant à un triangle équilatéral de 35-40 $\mathrm{m}$ de côté.

18. H. Mouillebouche, Les maisons fortes en Bourgogne du Nord du XIII au XVI siècle, Dijon, 2002, p. 216.

19. La comparaison avec des constructions datées similaires, la corniche à modillons, les ouvertures du dernier étage avec coussinets comparables à ceux du donjon de Semur-en-Auxois ou encore la fenêtre géminée du premier étage côté vallée.

20. C. Perrault, Châteauneuf-en-Auxois..., op. cit., p. 20.

21. De fortes présomptions font supposer la présence d'un logis antérieur à celui de la fin du $\mathrm{Xv}^{\mathrm{e}}$ siècle, notamment plusieurs reprises dans les élévations accessibles et visibles.

22. C. Perrault, Châteauneuf-en-Auxois..., op. cit., p. 20.

23. Catherine de Châteauneuf est condamnée au bûcher par le Parlement de Paris en 1456 pour avoir assassiné son époux avec un gâteau empoisonné afin de rejoindre Châteauneuf avec son amant.

24. J. P. C. LAVirotte, Annales de la ville d'Arnay-le-Duc en Bourgogne, Marseille, 1980 (rééd.), p. 151.

25. Voire de France: J. B uRnouf (dir.), Manuel d'archéologie médiévale et moderne, Paris, 2009, p. 275.

\section{AUTEUR}

\section{THOMAS VERGINE}

Centre Michel-de-Boüard / CRAHAM (UMR 6273), université de Caen-Normandie 